\title{
Editorial
}

\section{Coronary angioplasty is like going to the dentist}

The paper of Koch and colleagues in this issue takes advantage of a situation that is still commonplace in Holland but becomes quite rare in the rest of the world. ${ }^{1}$ Every year, thousands of Dutch patients are referred to invasive cardiac centres licensed to dilate by invasive cardiac centres barred from dilating by government ruling. Combining the therapeutic with the diagnostic act makes sense, is cost efficient, and patient friendly. It remains a taboo for the majority of Dutch invasive cardiologists working in so called diagnostic centres. While it is a taboo by legislation for them, it is a taboo by solidarity for their colleagues working in the few so called therapeutic centres. If they were combining the diagnostic angiogram with the coronary angioplasty in all their patients, their market advantage over their purely diagnostic colleagues would be so conspicuous that the usual competition between cardiac care providers would capsize for sure. The primary idea behind this system is to boost the quality of coronary angioplasty by having a large number of procedures performed in a small number of centres. Secondary ideas behind it are the cost concern that too many centres for cardiac surgery would be run just to chaperone the respective interventional cardiology activities and a certain desire to contain angioplasty numbers by setting up a road block on the way to the procedure.

A great majority of patients of the tertiary cardiac centre presenting their report on preselection of coronary angioplasty patients for short term observation ${ }^{1}$ were referred with a neat history, physical examination, and coronary angiogram from an invasive but non-interventional cardiac centre in the catchment area. About $90 \%$ of them subsequently underwent single vessel angioplasty which is in keeping with a contemporary European database. ${ }^{2}$ After stratifying them into those presumably not meaning trouble and those of some concern, roughly half of the overall angioplasty patients of the reporting centre remained for the study on the possibility of early discharge. The only exclusion criteria of note was unstable angina. It is not quite clear why an unstable situation should not qualify for early discharge, once the problem-that is, a single lesion in a perhaps not so important coronary artery-is identified and successfully treated by angioplasty.

Back transfer to the referring hospital was used as surrogate for early discharge. Although it is stated that clinical data were collected up to discharge from the referring hospital, some doubts may arise in the reader that data collection outside of the reporting hospital might not have been as thorough as inside. The patients were sent back to a variety of care systems, data collection protocols, and physician egos.

It is likely that it was not the foremost priority of the physicians in the referring hospitals to diligently look for, jot down, and report every problem that occurred in these patients up to their discharge. In particular, there was apparently no uniform protocol active for creatine kinase samples. Therefore, the number of infarctions cited for these patients has to be read with caution. Moreover, no meaningful mention is made about puncture site problems, the second most important complication after coronary angioplasty next to cardiac events.

The message of the paper still holds true. At least $50 \%$ (if not a great deal more) of a general coronary angioplasty population can be considered for outpatient treatment. In fewer than $10 \%$ of these patients, general wisdom imposed to convert the procedure to an inpatient procedure - that is, to keep the patient overnight. The majority of complications (in this study even all of them) will occur in the patients recognised in time as trouble makers. In the report discussed, every other of these patients was in fact fraught with at least one complication. All risk factors that were identified are known at the end of the procedure. At this point in time it can, therefore, be ordained whether the patient is set free or kept in. They were in order of importance acute (temporary) closure of the dilated vessel during the procedure, side branch occlusion, angioplasty failure, female sex, need for a stent to correct what can be subsumed under an "ugly" result, and bifurcation lesions.

It has to be regretted that the authors do not state whether the result of this study prompted them to follow their own recommendations and discharge the patients home rather than back to the referring hospital (a tremendous potential to save health expenditures), and whether they were willing to include unstable patients (about 30\% of their general angioplasty activity) in the same pattern of aftercare. They also missed out on harvesting highly relevant data offered during the procedure and partially already during the diagnostic coronary angiogram. First, completely occluded vessels (about $20 \%$ in their cohort) are extremely unlikely to cause trouble, unless a significant complication occurs during the intervention. ${ }^{3}$ Second, sufficient collateralisation seen on the diagnostic angiogram comes close to a guarantee that no significant clinical problem will occur, should the dilated vessel occlude abruptly. Third, collateralisation that may not be visible on the diagnostic study because the lesion is not tight enough may be tested easily during the procedure. If a balloon occlusion of several minutes is tolerated without pain or ECG changes, there are either sufficient collaterals or the respective myocardium is already infarcted. In both cases, an occlusion of the vessel is unlikely to cause any significant clinical event. Moreover, a contrast medium injection into the ipsilateral artery during balloon occlusion may show collaterals and provide the same information without having to extend the balloon occlusion for several minutes. If a flow wire or a pressure wire are used, valuable information about the degree of collateralisation and thus the degree of jeopardy in case of acute vessel occlusion can be gathered conveniently. ${ }^{4}$ 
The ultimate way to learn about collateralisation without additional hardware or time invested is what we call washout collaterometry. This entails injecting contrast medium into the vessel to be dilated and inflating the balloon simultaneously. The contrast medium caught distally to the balloon, the moment the vessel becomes completely occluded, either remains there as long as the balloon is occluding the vessel (no collaterals) or is washed out with a varying degree of rapidity which corresponds to the degree of collateralisation. The collaterals will be picked up irrespective of whether they originate from the ipsilateral or the contralateral vessel. This method is the only one that checks for all possible collaterals without need for additional catheters. If the dye caught distally is washed out within 10 cardiac beats, sufficient collateralisation to prevent a significant infarction in case of an acute vessel occlusion can be deduced. All these patients can probably be discharged the same day safely, regardless of whether or not a stent has been placed. A red flag has of course to be raised if additional problems occur, such as side branch occlusions which, notably, are about 10 times more frequent if a stent is used. Such a side branch may cause a small infarction and late ventricular fibrillation after the patient has been discharged home. It may also be the main contributor to the dilated vessel and fail to function if abrupt closure of the dilated vessel occurs after hospital discharge.

From the intriguing paper of Koch and colleagues, and from the above remarks, it can be concluded that coronary angioplasty is indeed like going to the dentist. In both procedures it is most attractive if diagnosis and treatment are done in a single session. The absence from home sweet home (and work for that matter) amounts to a few hours only, and there is no aftermath aside from a little soreness at the site the body was invaded (the mouth or the groin). There remains a difference, however. There is no sudden death from toothache or a filling coming loose. Hence, there is no way we can shift the entire angioplasty activity to the outpatient department. While it is clearly an overkill (actually the opposite of it) to monitor every angioplasty patient in the coronary care unit overnight, it is an overkill in the proper sense to send every patient home after the angioplasty procedure who does not show signs of an infarction already beginning. Using all the information provided plus the intraprocedure variables pointed out above, we should be able to identify about two thirds of elective angioplasty patients for a safe discharge on the day of the procedure. The concern of puncture site problems is virtually non-existent if the radial approach is used. ${ }^{5}$ With a limitation of 5000 units of heparin for all patients, which proved safe in this paper and in a prior randomised study, ${ }^{6}$ in conjunction with small guiding catheters and perhaps puncture site closure devices, a femoral approach with an observation period of about four hours should be safe, too.

We should not fall for the idea that pushing the stenting rate from $<30 \%$ in this paper to $>80 \%$ as featured at many institutions currently, will make coronary angioplasty even safer. The trough of cardiac complications is probably reached at a stenting rate of $30-50 \%$. The remainder of stents are implanted to prevent restenosis but re-augment the risk for acute occlusion (particularly side branch closures) to a level that may be even higher than that before the stent era.

Before taking the drastic step of rationing access to coronary angioplasty for cost containment, other possibilities to save money have to be fully exploited. Foremost among them are the combination of diagnostic and therapeutic interventions (the rule for the dentist) and short hospital stays. The slogan should be "in and out the same day, back to work the next day, just like going to the dentist".

BERNHARD MEIER

Cardiovascular Department, University Hospital,

CH-3010 Bern, Switzerland

email:bernhard.meier@insel.ch

1 Koch KT, Piek JJ, Prins MH, De Winter RJ, et al. Triage of patients for short-term observation after elective coronary angioplasty. Heart 2000;83:557-63.

2 Maier W, Enderlin MF, Bonzel T, et al. Audit and quality control in angioplasty in Europe: procedural results of the AQUA Study 1997: assessment of 250 randomly selected coronary interventions performed in 25 centres of five European countries. AQUA study group, nucleus clinical issues, working group coronary circulation, of the European Society of Cardiology. Eur Heart f 1999;20:1261-70.

3 Meier B. Total coronary occlusion: a different animal? $\mathcal{F}$ Am Coll Cardiol 1991;17:50B-7B

4 Seiler C, Fleisch M, Garachemani A, Meier B. Coronary collateral quantitation in patients with coronary artery disease using intravascular flow velocity or pressure measurements. $\mathcal{F}$ Am Coll Cardiol 1998;32:1272-9.

5 Kiemeneij F, Laarman GJ, Odekerken D, et al. A randomized comparison of percutaneous transluminal coronary angioplasty by the radial, brachial, and percutaneous transluminal coronary angioplasty by the rad
femoral approaches. $7 \mathrm{Am}$ Coll Cardiol 1997;31:1269-75.

6 Vainer J, Fleisch M, Gunnes P, et al. Low dose heparin for routine coronary angioplasty and stenting. Am F Cardiol 1996;78:964-6. 